\title{
Dextran Sulfate Based Gel Coating for Drug Eluting Balloon Angioplasty
}

\author{
Jenna Mosier*, Hannah Stealey, Kalifa Stringfield, Katie Webb, \& C. LaShan Simpson \\ Department of Agricultural and Biological Engineering, Mississippi State University, Mississippi State, MS \\ Students: jmosier243@gmail.com*, bannahstealey19@gmail.com,kalifastringfield@gmail.com, katiewebb1212@gmail.com \\ Mentor: clsimpson@abe.msstate.edu
}

\begin{abstract}
Vascular calcification, a consequence of cardiovascular disease, disrupts natural blood flow and can result in death. Common treatment efforts include various anti-inflammatory medications, balloon angioplasty, or stents, with little success in completely reversing calcification. The proposed design focuses on improving current drug-eluting stents by developing a dextran-sulfatebased gel drug delivery system loaded with receptor activator of nuclear kappa B-ligand (RANKL) to induce osteoclast differentiation. To ensure that the gel could adequately deliver RANKL, the gel was tested for its affinity for hydroxyapatite (HA), a critical component of calcification, and its ability to withstand shear. Infrared spectroscopy (IR) indicated binding to HA. Preliminary scanning electron microscopy and energy dispersive X-ray spectroscopy (SEM/EDS) results confirmed the presence of calcium on the gel after a one-hour soak in an HA mixture. Shear testing demonstrated that negligible protein, an average of $0.029 \pm 0.024 \mu \mathrm{g} / \mathrm{mL}$, was sheared off under flow conditions, indicating that the gel is stable for the duration of balloon delivery. These preliminary results indicate that a dextran-sulfate-based gel has the potential to serve as a therapeutic gel-coating to treat vascular calcification. Future experimentation will include a co-culture study to determine whether osteoclast progenitor cells will adequately proliferate and differentiate in the presence of the RANKL-loaded gel.
\end{abstract}

\section{KEYWORDS}

Angioplasty; Calcification; Cardiovascular; Dextran; Gel; Osteoclast; Stent; Vascular

\section{INTRODUCTION}

Cardiovascular disease is one of the most prevalent problems in the United States, causing one in three deaths annually. ${ }^{1}$ This condition can be manifested in various forms: heart disease, hypertension, diabetes, and many more. One particular consequence is vascular calcification. Smooth muscle cells lining the interior walls and in the medial layer of the artery are not terminally differentiated, meaning they can mature into different cell types. When calcium and phosphate levels increase in the bloodstream due to cardiovascular disease, they trigger a response in the tissue, causing arterial cells to differentiate into atypical phenotypes and deposit bone-like tissue. ${ }^{2}$ This active transformation of healthy tissue into diseased tissue along the lining of the artery causes the vasculature to stiffen and calcify, disrupting natural, laminar blood flow. ${ }^{3}$ The calcification prevents the arteries from dilating and constricting naturally and prevents blood flow through the lumen. The plaque buildup is living, inflamed tissue that continues to expand when aggravated. ${ }^{4}$

A current method used to treat vascular calcification is balloon angioplasty. During this procedure, a catheter is inserted through the femoral artery and fed to the site of calcification in the vasculature. A balloon is attached to the end of the catheter, typically one to five $\mathrm{mm}$ in diameter. ${ }^{5}$ Once in place, the balloon is inflated so that the force from deployment breaks up the calcification and clears the bloodstream. While this is temporarily successful, the recently disturbed tissue recruits more inflammatory molecules and restenosis, the return of plaque buildup, occurs. ${ }^{4}$ One way to counteract this issue is using an antiinflammatory agent to coat the balloon that can be released upon deployment. This design was first published in the 1980s, using the drug Paclitaxel as a counter-agent to inflammation. ${ }^{6}$ While it prolongs the time before restenosis occurs in the arteries; the use of Paclitaxel still leads to significant scar tissue and irreversible damage to the artery. ${ }^{7}$

Despite the downfalls associated with Paclitaxel, the drug-eluting balloon is still an effective design for drug delivery to vascular calcification. A gel coating could be employed to release a specific drug at a predetermined concentration slowly. The gel would need to be soluble, non-toxic, biocompatible, and able to bind to hydroxyapatite $\left(\mathrm{Ca}_{5}\left(\mathrm{PO}_{4}\right)_{3}\right)(\mathrm{HA})$, the main component of calcification in the arteries. ${ }^{8}$ Dextran-sulfate sodium salt is a previously used gel base that effectively covers these categories. ${ }^{9}$ Dextran is soluble in water and nontoxic, making it a viable option. Due to its hydrophilic nature, enhanced when combined with sulfate, dextran was studied for use as a delivery mechanism for hydrophobic, therapeutic drugs in the body.9,10 It has shown success as a microsphere for drug delivery without losing important cargo before it reaches its desired location within the body. Clinically, it has been used as a therapeutic agent in various ways, including increasing plasma expansion and peripheral blood flow. ${ }^{11}$ At a low molecular weight, it has a typical half-life of eight hours, whereas its higher molecular weight counterparts exhibit an even longer half-life, making it an ideal vehicle for slow release. ${ }^{11,12}$ Dextran-sulfate has been previously used to form nanospheres loaded with insulin for delivery. ${ }^{13}$ As a hydrogel, it has been studied extensively as a delivery mechanism, with water 
content playing a significant role in the stability of the gel. ${ }^{14}$ In this study, we aim to show how this dextran-based gel is suitable for anti-calcification agent delivery due to its ability to bind to calcium deposits and withstand the shear force induced by blood flow.

The RANK/RANKL/OPG pathway is an important consideration in choosing a drug to load into the gel for delivery. While the pathway is mostly understood according to current research, conflicting reports on its effects in bone versus vasculature make it difficult to determine its exact mechanism of action. It is known that increased serum osteoprotegerin (OPG) is related to a decrease in bone loss and osteoclast activation in the skeletal system. ${ }^{15}$ OPG blocks RANK receptors on monocytes and macrophages in bone and prevents their differentiation into bone-resorbing bodies. This is where the gene, RANKL, becomes important. It naturally binds to the RANK receptors when uninhibited by OPG and initiates the differentiation process. RANKL upregulation is associated with an increase in osteoclastogenesis, which initiates the formation of osteoclasts. These cells then begin remodeling bone, leading to an increased bone loss. ${ }^{16,17} \mathrm{It}$ is hypothesized that RANKL could be introduced to the vasculature to activate monocytes and macrophages, causing differentiation into osteoclasts to resorb the plaque present in the arterial wall.

However, it is important to note that further exploration of the relationship of OPG and RANKL expression in the vascular system reveals that RANKL may be associated with plaque rupture in unstable coronary artery disease. ${ }^{18}$ Similarly, OPG, which plays a role in blocking osteoclastogenesis in bone, has been theorized to play a protective role against vascular calcification. ${ }^{19}$ In a study where OPG was knocked down in transgenic mice, an increased onset of vascular calcification was observed. ${ }^{19}$ In a different study measuring levels of OPG and RANKL in uremic mice, it was found that increased expression of RANKL was correlated with plaque accumulation in the arteries and increased observation of vascular calcification. ${ }^{20}$

This paradoxical relationship of OPG and RANKL to bone resorption is poorly understood. It is well known that vascular calcification and bone resorption are linked; an increase in bone loss causes an increase in bone formation in the arteries, and the opposite is true. The same factors that decrease bone mass in the skeletal system appear to increase bone mass in the arteries. As previously mentioned, this has been shown through the upregulation of RANKL in vascular calcification. ${ }^{18}$ However, there are two potential explanations for this issue. First, it is important to recognize that RANKL is currently viewed as a biomarker rather than a mediator. Increased RANKL is triggered by the aggregation of inflammatory molecules and cytokines in order to maintain balance through an immune response. ${ }^{21}$ Because inflammatory molecules are recruited during calcification; it is natural to see an increase in the recruitment of RANKL to help ameliorate this issue. This could account for the increase in RANKL expression.

Secondly, OPG is found in two primary locations: both in the serum and the plasma. High concentrations in each can lead to significantly different outcomes. An increase in serum OPG is reported to lead to bone destabilization and breakdown, while an increase in plasma OPG is associated with increased atherosclerosis. ${ }^{21}$ Fibrinogen, a clotting factor found in plasma, could be a component at play in this observation, meaning that the location of delivery is a determining factor in the effects of the gene.

Through this investigation, we believe that RANKL has the potential to reverse arterial calcification if delivered directly to the site of injury using the modified dextran-sulfate gel model. ${ }^{9}$ To further explore this potential treatment, our first goal was to determine the effectiveness of dextran-sulfate as a gel vehicle for RANKL delivery by using the protein bovine serum albumin (BSA). BSA was used as a substitute for RANKL because they are both soluble and have similar molecular weights (BSA averaging $\sim 50-60 \mathrm{kDa}$ and RANKL $\sim 60-70 \mathrm{kDa}$ ) (Sigma). ${ }^{22}$ These molecular weights, while not exact, are similar enough to validate using BSA as a RANKL surrogate. BSA is soluble in ethanol and glycerol, making it suitable for testing purposes. Finally, it is a readily available substitute that can be measured using the described methods. In this study, we looked at two separate aspects of the gel: 1) its ability to effectively bind to HA to determine whether it would stay in place once delivered to calcification and 2) its ability to withstand the shear forces incurred by blood flow in the artery without completely dissolving before drug (RANKL) could be delivered. Here, we use the dextran-sulfate based gel cast on nylon and incorporate BSA as a substitute for RANKL. With the use of IR spectrometry, SEM and EDS, and shear flow testing, we examined binding properties. Our results indicate future potential success for this application.

\section{METHODS AND PROCEDURES}

All materials were purchased from Sigma Aldrich unless otherwise noted.

\section{Gel Formation}

The gel formation protocol was developed as a modified model from Lamichhane. ${ }^{9}$ In short, 1.875 g of dextran sodium sulfate salt, $2.25 \mathrm{~mL}$ distilled water, and $0.38 \mathrm{~mL}$ glycerin were combined on a stir plate at $200 \mathrm{RPM}$ for three hours. If no BSA was used, $1 \mathrm{~mL}$ of ethanol was added to the mixture while on the stir plate. For experiments loaded with BSA, $65.55 \mathrm{mg}$ BSA was added to the mixture while on the stir plate, allowed to dissolve, and then followed by $1 \mathrm{~mL}$ of ethanol. The gel continued to stir for 30 minutes at room temperature. Once combined, the gel was spread evenly in a thick layer (0.1-0.5 mm thick) over either a thin sheet of sterile nylon (purchased from VWR) or catheter tubing (donated by Oktibbeha County Hospital), dependent on testing. 
Gels were cross-linked to increase surface stability and lifespan. To do so, gels were dipped in a 1:9 ratio of glycerin to ethanol mixture for $30 \mathrm{~s}$ each followed by dipping in 100\% ethanol for one minute. A setting period of 24 hours elapsed for each gel before additional testing to allow gel time to dry.

\section{Hydroxyapatite Coating.}

To determine binding ability to HA, gels formed on nylon sheets $(2 \mathrm{~cm} \times 2 \mathrm{~cm})$ were placed in beakers with $1 \mathrm{X}$ Phosphate Buffered Saline (PBS) and $15 \mu \mathrm{M} \mathrm{HA}$ in a $37^{\circ} \mathrm{C}$ shaker to mimic in vivo conditions. Gels soaked for either one hour, four hours, or 24 hours with agitation. Gels were then rinsed in fresh 1X PBS and left to dry for a minimum of 24 hours before testing.

\section{IR Spectroscopy}

The gel samples previously soaked in HA for one hour, four hours, and 24 hours were tested with infrared spectroscopy (IR) using MicroLab technology to measure absorbance and transmittance of the sample. A control sample, dextran gel that was not soaked in the HA/PBS mixture, was tested for baseline reference, as well as pure powdered HA. All samples soaked in the HA/PBS mixture were dried for at least 24 hours to ensure that no water was left in the sample to affect the IR reading. For sample preparation, the gel was carefully removed from nylon after being dried, then directly transferred to the crystal for analysis. After the machine-generated graphs for each sample, peaks were analyzed and classified using a standard table of wavelengths.

\section{Scanning Electron Microscopy/Energy Dispersive Spectroscopy}

The same gel sample preparation procedure previously described for IR analysis (gels soaked in HA for one hour, four hours, and 24 hours) was also used to prepare samples for analysis with SEM and EDS testing. Small squares $(1 \mathrm{~cm} \times 1 \mathrm{~cm})$ were cut from the center of the gels on the nylon sheets to obtain the most representative sample. Samples were then mounted on a metal peg and coated with a $15 \mathrm{~nm}$ thick layer of platinum. A JEOL 6500F Field Emission SEM with an X-EDS spectrometer and Oxford Instruments INCAEnergy+ software was used for analysis.

\section{Shear Testing}

An expired standard hospital catheter $(4.36 \mathrm{~mm}$ diameter) was modified for use in recapitulating blood flow through an artery. Two experimental designs were used to test the adhesion of BSA to the gel. Dulbecco's Modified Eagle's Medium (DMEM) was used to mimic blood, as is standard in blood shear flow testing. A syringe pump (Fisher Scientific) was paired with a syringe containing DMEM, as shown in Figure 1. Catheter tubing was cut to $10 \mathrm{~cm}$, and the gel was placed opposite of end connected to the syringe. A flow rate of $956.9 \mathrm{~mL} / \mathrm{hr}$ created a shear force on the wall of the tubing as well as the catheter surface. The flow rate was chosen based on a published model of in vitro blood flow (see Discussion). ${ }^{23}$ Runoff was collected in a $100 \mathrm{~mL}$ beaker and briefly disturbed by shaking before aliquots were taken to ensure even sample collection. Aliquots of $1 \mathrm{~mL}$ were taken at the following time points for BSA analysis: 30 seconds and every minute for six minutes. This process was repeated twice.

The process was repeated a third time with an alternative design. The same tubing was used; however, a coated catheter was not used. Instead, a microscope slide with a small square sheet of nylon coated with the gel was placed diagonally into a beaker, and the same flow rate and syringe set-up were used as outlined previously. Aliquots of $1 \mathrm{~mL}$ were taken at the following time points for BSA analysis: 30 seconds, and every minute for six minutes.

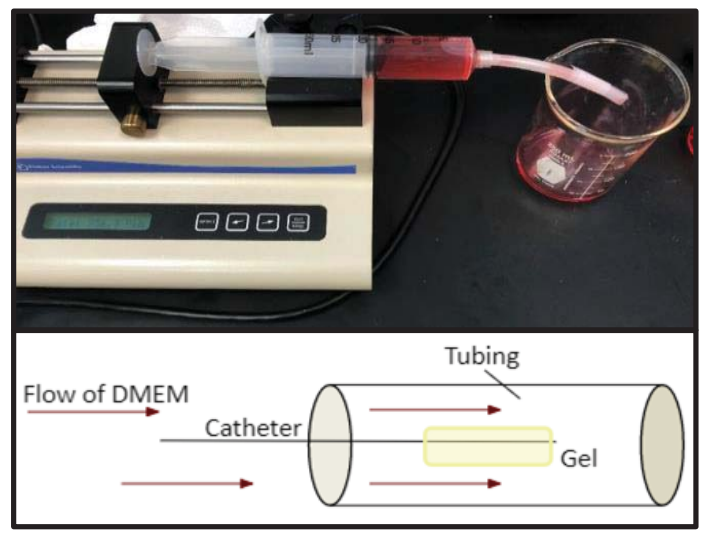

Figure 1. The shear flow protocol is illustrated where the gel coated catheter is fed through plastic tubing and connecting to a syringe pump. 


\section{BCA Assay}

A bicinchoninic acid (BCA) assay was used to determine the concentration of protein sheared off during the shear testing. Reagents were prepared according to the kit (Thermofisher). In a standard 96-well plate, $50 \mu \mathrm{L}$ was transferred from each time point aliquot in triplicate. A standard concentration curve was established, and the 96-well plate was read using a spectrophotometer. Results were analyzed by comparing samples to the standard curve.

\section{RESULTS}

Binding Assessment

IR produced spectra (transmittance vs. wavelength) that provided information about chemical bonds. Two control samples, dextran gel only, without being soaked in HA/PBS mixture, and HA only, in powdered form, were read. As previously mentioned, samples were prepared by removing the cast gel from the nylon after soaking in HA/PBS for the allotted time, dried for 24 hours, and placed directly on the machine's crystal for analysis. In Figure 2 depicting IR results from all samples, the control tests revealed an aromatic carbon-carbon double bond visible around $1600 \mathrm{~cm}^{-1}$, from the ring characteristic of dextran, as well as carbon-hydrogen bonds appearing in the $2900 \mathrm{~cm}^{-1}$ region. No peak occurs at $1200 \mathrm{~cm}^{-1}$. In the HA only test, the presence of a potential phosphate $\left(\mathrm{PO}_{4}\right)$ bond appears at $500 \mathrm{~cm}^{-1}$. No peaks occur at the $1200 \mathrm{~cm}^{-1}$ and $3300 \mathrm{~cm}^{-1}$ wavelengths.

A sample from each of the PBS/HA soak time points (one hour, four hours, and 24 hours) was tested using IR. A small amount of gel remained at the four hour and 24-hour time points. For the one hour, dip-coated gel, peaks can be seen starting to form at $1200 \mathrm{~cm}^{-1}$ and $3300 \mathrm{~cm}^{-1}$. For the four hour PBS/HA soak of the dip-coated gel, peaks can be observed surrounding the $500 \mathrm{~cm}^{-1}$ and around the $1500 \mathrm{~cm}^{-1}$ region. Additionally, peaks can be seen beginning to form around the $3300 \mathrm{~cm}^{-1} \mathrm{mark}$. A notable peak appears around the $500 \mathrm{~cm}^{-1}$ mark. A peak can be seen beginning to form at the $1200 \mathrm{~cm}^{-1}$ mark.

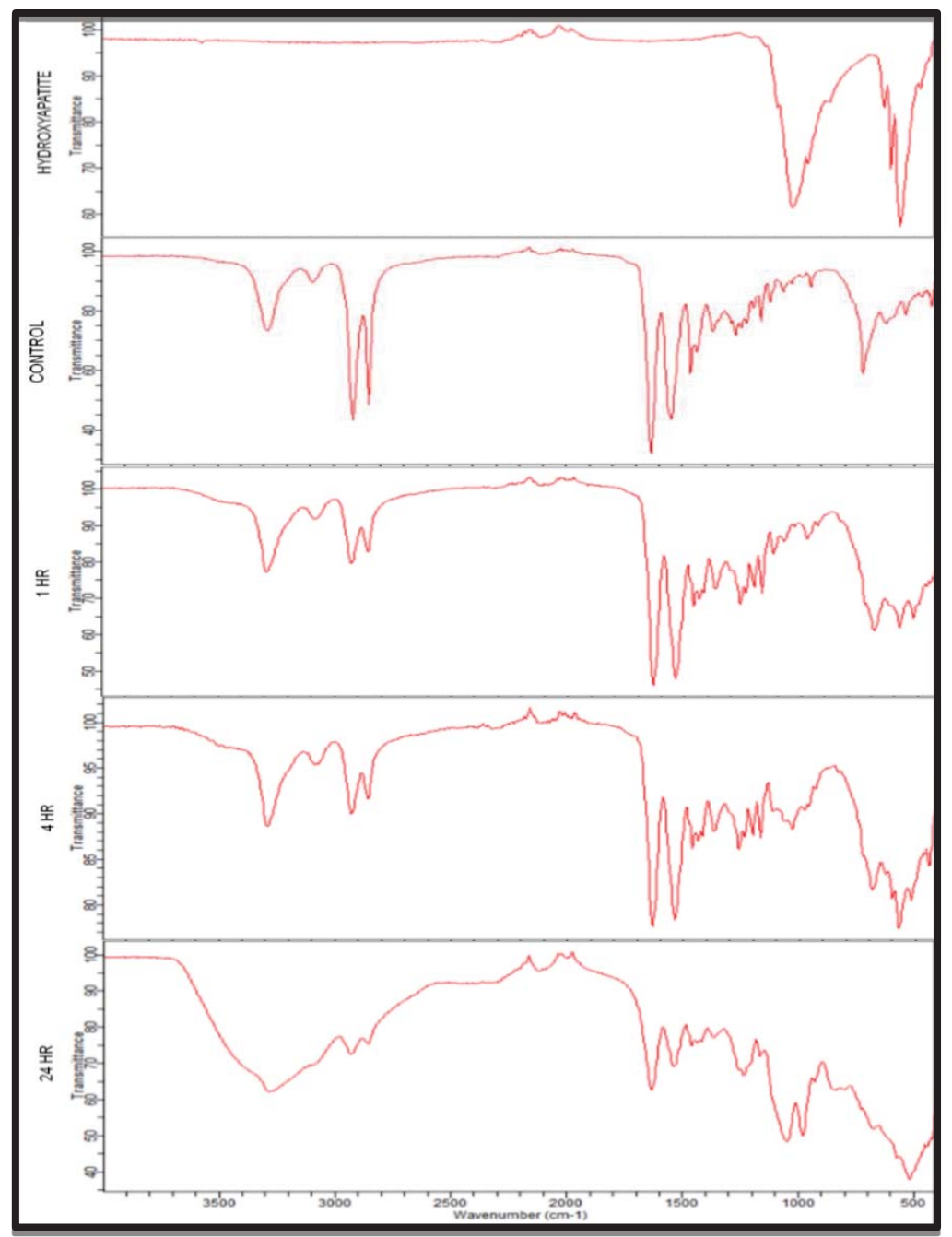

Figure 2. IR spectra results for HA only, control, one, four, and 24-hour gels. Increase in intensity at $1200 \mathrm{~cm}^{-1}$ indicates Ca binding to $\mathrm{SO}_{4}$. 


\section{Shear Force Assessment}

A BCA assay revealed the protein concentration that was released from the gel on the catheter during shear force testing. The assay was completed in triplicate, and the average value for each of the three trials was averaged together. Effectively no protein (BSA) was sheared off between the zero minute and five-minute time points. Figure 3 depicts the concentration of protein in $\mu \mathrm{g} / \mathrm{mL}$ in the media collected for aliquots at each time point (minutes). Between the five to six minute time period, an average of $0.029 \pm 0.024 \mu \mathrm{g} / \mathrm{mL}$ was released from the gel.

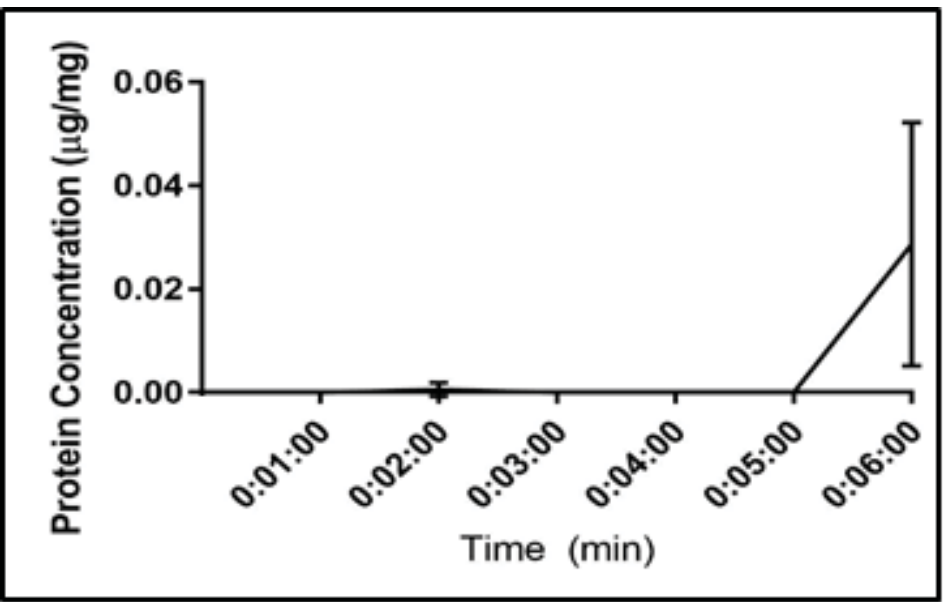

Figure 3. The graph above illustrates the results from shear testing, with $0.55 \pm 1.3 \mathrm{ng} / \mathrm{mL}$ released at two minutes, and $0.029 \pm 0.024 \mu \mathrm{g} / \mathrm{mL}$ released from five to six minutes.

When the end of the catheter was coated in BSA loaded gel and used for shear flow testing, minimal protein was lost between the zero and one minute mark. Similar results were seen with the slant test as described above, in which $0.1184 \pm 0.049 \mu \mathrm{g} / \mathrm{mL} \mathrm{of}$ protein was found in collected media at the five to six-minute mark.

Scanning Electron Microscopy (SEM)/Energy Dispersive Spectrometry (EDS) Results

The one hour sample produced definitive results, shown in Figure 4, which calcium was indeed found on the surface of the gel. After soaking one hour in PBS/HA, calcium appeared on the sample at $0.45 \%$ by weight, with the images shown below taken at the $100 \mu \mathrm{m}$ scale. The four hour and 24-hour samples produced negligible calcium content by EDS measurement.
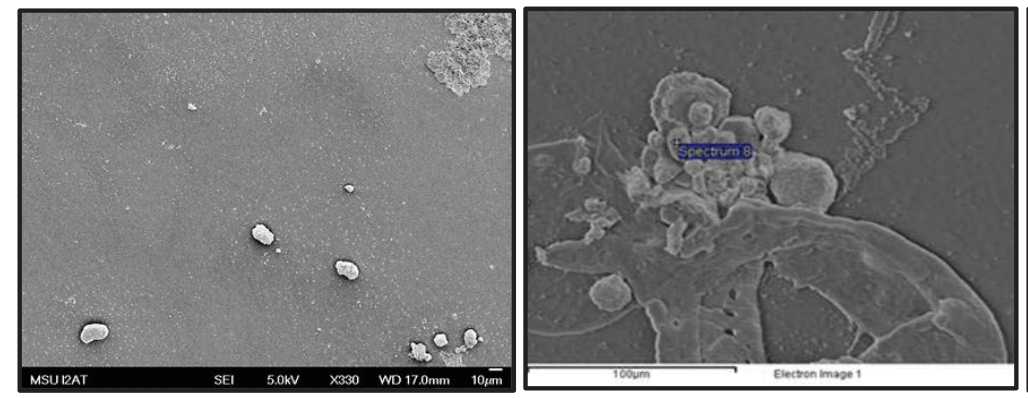

\begin{tabular}{|c|c|c|}
\hline Element & Weight\% & Atomic\% \\
\hline $\mathrm{C}$ & 82.58 & 87.69 \\
\hline $\mathrm{O}$ & 13.88 & 11.07 \\
\hline $\mathrm{P}$ & 0.15 & 0.06 \\
\hline $\mathrm{Ca}$ & 0.45 & 0.14 \\
\hline
\end{tabular}

Figure 4. EDS testing revealed that the calcium on the surface of the gel was $0.45 \%$ by weight.

\section{DISCUSSION}

\section{Design criteria}

While current treatments are available to help reduce the effect of calcification on blood flow, no treatment completely eradicates vascular calcification. As a result, subsequent interventions are needed. The concept for these constructs is founded upon integral components that work in tandem: a gel coated balloon angioplasty procedure that can deliver anti-calcification agents to the vessel. The gel coating should detach from the balloon and adhere to calcification in order to time-release a concentration of RANKL. Once applied to the site of vascular calcification, the RANKL should combat the hydroxyapatite buildup by causing macrophage differentiation into osteoclasts to resorb bone tissue. 
The gel coating requirements included the following: non-toxicity, biocompatibility, cost-effectiveness, and stability in aqueous environments, ability to bind to hydroxyapatite and carry a therapeutic drug to the site of calcification. Previous use of dextran-sulfate gel has also confirmed its hydrophilic and biocompatible properties make it useful as a drug delivery mechanism. ${ }^{9}$ However, with our results, we can show that it can bind in the desired manner to sites of calcification where RANKL delivery will be most useful. We also show that it can withstand high shear forces without losing loaded protein too quickly.

\section{Comparison of gel-to-nylon coating methods}

Two coating methods were tested in the early stages of this experiment: spin-coating and dip-coating. The dip-coating method on the thin nylon film pieces is commonly used with sol-gels and allowed for a thicker layer of gel to be formed. ${ }^{24}$ Conversely, spincoating resulted in a thin, even layer on nylon. After the mixture of glycerol and ethanol was used to cross-link the spun gel, it dissolved nearly instantaneously, too quickly for practical purposes. As well, if this design were to be mass-produced, a dip coating may be a more cost-effective approach, as no specialized machinery would be required. Sol-gel dip-coating methods have been reported as inexpensive and easier to employ than spray or sputter-coatings. ${ }^{24-26}$ Because the dip-coating method can be applied independent of the shape of the object being coated, it allows for a uniform coating. ${ }^{25}$

\section{Roles of dextran, BSA, and bydroxyapatite}

Dextran-sulfate sodium salt provides a non-toxic, water-soluble, and relatively inexpensive vehicle for drug delivery. ${ }^{10}$ For preliminary testing purposes, BSA was chosen to represent RANKL because of its similarity in molecular weight. Our design involves targeting and attachment to sites of arterial calcification. To model this in vitro, the main component of these calcium aggregates, HA, was used to assess the gel's targeted binding capabilities. To our knowledge, dextran-sulfate has not been previously studied concerning binding to either bone material or hydroxyapatite, though multiple studies have examined hydrogels as potential scaffolds for bone regeneration. ${ }^{27,28}$ Many gels have also been functionalized with different peptides in previous experiments to be used as bone defect fillers. ${ }^{29}$

\section{IR Testing}

The most significant results achieved were those at the one-hour PBS/HA soak time point. Referring to Figure 5, peaks can be seen starting to form at $1200 \mathrm{~cm}^{-1}$, indicating calcium binding to sulfate. This signifies that the dextran-based gel has successfully bound to HA. Additionally, the peak at $3300 \mathrm{~cm}^{-1}$ corresponds with hydroxyl $(-\mathrm{OH})$ groups present after binding. Peaks at approximately $1000 \mathrm{~cm}^{-1}$ are characteristic of HA. Intensity decreases at the $2900 \mathrm{~cm}^{-1}$ peak (C-H bond) which could indicate the loss of $\mathrm{C}-\mathrm{H}$ bonds where $\mathrm{Ca}_{3}\left(\mathrm{PO}_{4}\right)_{2}$ is binding.

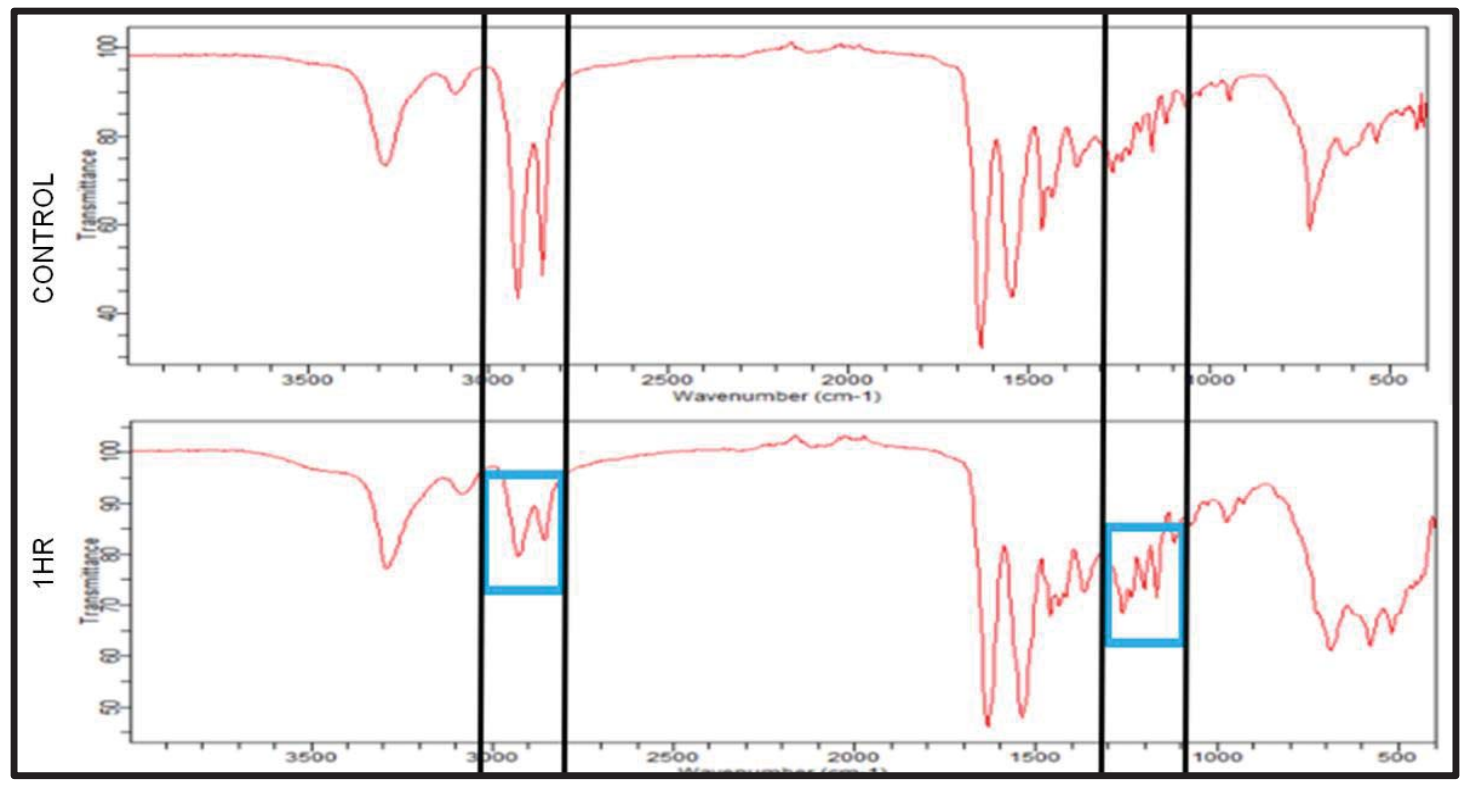

Figure 5. After one hour, a peak forms at $1200 \mathrm{~cm}^{-1}$, indicating calcium binding with sulfate (HA to gel). There is a loss of intensity at $2900 \mathrm{~cm}-1$ (C-H bond) that could indicate the loss of $\mathrm{C}-\mathrm{H}$ bonds where $\mathrm{Ca}_{3}\left(\mathrm{PO}_{4}\right)_{2}$ is binding 


\section{SEM/EDS Testing}

Preliminary SEM and EDS results in Figure $\mathbf{6}$ provide evidence that calcium was present on the dip-coated sample that was soaked in the HA/PBS solution for one hour. Ideally, this means that the gel can adhere to calcification for at least one hour. However, the one and 24-hour samples show no signs of calcium still on the surface of the gel. This is potentially the result of the gel dissolving over the longer period of time in solution. From this, it is hypothesized that the gel would be able to bind to the calcium deposits for the duration of time needed for the balloon to be inflated, removed, and then allow for the release of RANKL at the site. Further testing is required to confirm this hypothesis.
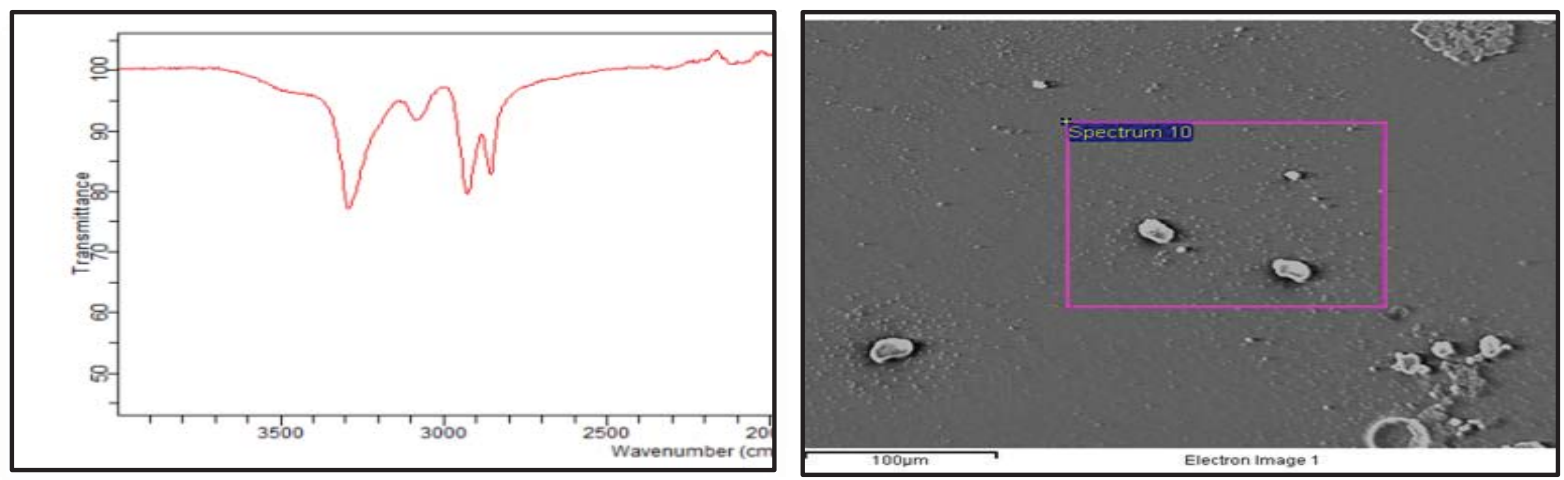

Figure 6. IR for the one-hour soaked gel confirmed through SEM imaging with evidence of Calcium bound to the gel surface.

\section{Shear Testing}

Though blood flow and shear stress on arterial walls proves a contentious topic in bio-fluid mechanics, a previous study shows a validated computational model with a flow rate of $19.4 \mathrm{~mL} / \mathrm{min}$ (or 1,164 mL/hr). ${ }^{23}$ This number was decided on using a pulsatile flow equation, shown in Equation 1 below using a dynamic blood viscosity of $1.5 \mathrm{cP}$. Because DMEM has a lower viscosity (1.1 $\mathrm{cP}$ ), a lower speed is required to simulate blood flow across the same cross-sectional area. The accepted blood flow rate according to Engel et al. is $19.4 \mathrm{~mL} / \mathrm{min}^{23}$ The max speed on the machine used was $\sim 16 \mathrm{~mL} / \mathrm{min}$ and was therefore used for shear testing.

$$
u(r, t)=\operatorname{Real}\left(\sum_{\omega=1}^{\infty} \frac{\widehat{q}}{\pi R^{2}}\left(\frac{\left(1-\frac{J_{0}\left(\alpha \frac{r}{R} i^{3 / 2}\right)}{J_{0}\left(\alpha i^{3 / 2}\right)}\right)}{\left(1-\frac{2 J_{1}\left(\alpha i^{3 / 2}\right)}{\alpha i^{3 / 2} J_{0}\left(\alpha i^{3 / 2}\right)}\right)}\right) e^{i \omega t}\right),
$$

Equation 1.

While it is difficult to narrow down a specific value for blood flow through an artery, for this preliminary study, a rate between 15 $20 \mathrm{~mL} /$ min most accurately reflects what can be done outside of in vivo testing according to literature. ${ }^{30}$ The speed is large enough to simulate the transitional blood flow moving through a vessel with a diameter of three to five $\mathrm{mm}$.

From the quantitative measurement of protein in shared media, the dextran-based gel appears to be successful regarding a delivery vehicle for anti-calcification agents. Ideally, the gel should hold the medication or drug long enough for the balloon to reach the site of calcification in the arteries. According to reports on angioplasty procedures, the standard time for a surgeon to deliver the balloon is three to five minutes. ${ }^{31}$ From shear testing, it was found that little to no protein was lost in the first five minutes of shear flow over the gel. Once the five-minute mark was reached, minimal amounts of BSA appeared in the collected media. This is indicative of proper release time in terms of the gel dissolving to allow RANKL to bind to calcification. However, further testing is required to ensure that RANKL would behave similarly. In previous studies, RANKL and fluorescent dextran were incubated together with ST2 cells and were shown to co-localize together in the same regions within the cells, indicating that they may have similar binding affinities. ${ }^{32}$

\section{CONCLUSIONS}

While a drug-eluting stent is already available in commercial use, it is not wholly effective due to design elements and drug choice. The current drug is Paclitaxel; however, it is not an adequate countermeasure to calcification and staunches the magnitude of the impacts. ${ }^{7}$ While future work is required to explore the success of RANKL over Paclitaxel, the current experimentation sought to 
determine whether a dextran-based gel could effectively bind to calcification sites and deliver RANKL. Three distinct assertions were formed in investigating the gel's properties. Dextran-sulfate sodium salt lent a satisfactory outcome as a binding agent. The gel was able to endure the hydroxyapatite soak treatments. The dextran-sulfate and HA were able to adhere to one another, signifying favorable attachment. The dextran-sulfate gel was able to withstand the in vivo shear forces of the blood that it would encounter during the surgical procedure of balloon angioplasty. Future implications for the work done indicate the need for further testing at a more complex level. This would involve replacing BSA with RANKL to determine if it exhibits similar behavior. Explicitly, a re-evaluation would be beneficial to solidify that the conclusions drawn from the research project are accredited. The outcomes from the SEM and EDS supplied qualitative answers and confirmed the IR results. However, a verification of these experimental developments is necessary. As well, a co-culture involving osteoclast progenitor cells in the presence of the gel would allow observation of whether or not cells will both survive and differentiate in the presence of the drug. An in vivo assessment of shear flow is also required to prove the blood flow model presently used accurately reflects working conditions and to give evidence to the overall success of the design.

\section{ACKNOWLEDGMENTS}

Authors thank Dr. Yu in the Agricultural and Biological Engineering Department at Mississippi State University for guidance throughout the project. Authors also thank Dr. Scott and Guerry Sanchez in the chemistry department for the use of IR instrument. Authors also thank the previous senior design group, notably Christian Griffith and Rachel Hybart for their preliminary research and guidance.

\section{REFERENCES}

1. Benjamin, EJ., Blaha, MJ., Chiuve, SE., Cushman, M., Das, SR., Deo, R., de Ferranti, SD., Floyd, J., Fornage, M., Gillespie, C., Isasi, CR., Jimenez, MC., Jordan, LC., Judd, SE., Lackland, D., Lichtman, JH., Lisabeth, L., Liu, S., Longenecker, CT., Mackey, RH., Matsushita, K., Mozaffarian, D., Mussolino, ME., Nasir, K., Neumar, RW., Palaniappan, L., Pandey, DK., Thiagarajan, RR., Reeves, MJ., Ritchey, M., Rodriguez, CJ., Roth, GA., Rosamond, WD., Sasson, C., Towfighi, A., Tsao, CW., Turner, MB., Virani, SS., Voeks, JH., Willey, JZ., Wilkins, JT., Wu, JHY., Alger, HM., Wong, SS., Muntner, P. (2017). Heart Disease and Stroke Statistics 2017 At-a-Glance. Circulation, n.d. https:/ / healthmetrics.heart.org/wpcontent/ uploads/2017/06/Heart-Disease-and-Stroke-Statistics-2017-ucm_491265.pdf

2. Lee, KM., Lee, EO., Lee, YR., Joo, HK., Park, MS., Kim, C., Choi, S., Jeong, JO., and Jeon, BH. (2017). APE1/Ref-1 Inhibits Phosphate-Induced Calcification and Osteoblastic Phenotype Changes in Vascular Smooth Muscle Cells. IJMS, 18(10), 2053. https://doi.org/10.3390/ijms18102053

3. Torremadé, N., Bozic, M., Panizo, S., Barrio-Vazquez, S., Fernandez-Martín, JL., Encinas, M., Goltzman, D., Arcidiacono, MV., Fernandez, E., Valdivielso, JM. (2016). Vascular Calcification Induced by Chronic Kidney Disease Is Mediated by an Increase of $1 \alpha$-Hydroxylase Expression in Vascular Smooth Muscle Cells. Journal of Bone and Mineral Research. bttps://doi.org/10.1002/jbmr.2852

4. Redfors, B., Maehara, A., Witzenbichler, B., Weisz, G., Stuckey, TD., Henry, TD., McAndrew, T., Mehran, R., Kirtane, AJ., Stone, GW., Généreux, P. (2017). Outcomes After Successful Percutaneous Coronary Intervention of Calcified Lesions Using Rotational Atherectomy, Cutting Balloon Angioplasty, or Balloon-Only Angioplasty Before Drug-Eluting Stent Implantation. J of Invasive Cardiol, 11, 378-386.

5. Medtronic. Coronary Balloons, (2011). http://wnw.medtronic.com/us-en/healthcare-professionals/products/cardiovascular/coronaryballoons.html (accessed January 2018)

6. Iqbal, J., Gunn, J., Serruys, PW. (2013). Coronary stents: historical development, current status, and future directions. British Medical Bulletin, 106(1), 193-211. https:/ / doi.org/10.1093/ bmb/ldt009

7. Cohen, JL., Glickstein, JS., Crystal, MA. (2017). Drug-Coated Balloon Angioplasty: A Novel Treatment for Pulmonary Artery In-Stent Stenosis in a Patient with Williams Syndrome. Pediatric Cardiology, 38(8), 1716-1721. bttps://doi.org/10.1007/s00246017-1646-1

8. Richards, JM., Kunitake, J., Hunt, HB., Wnorowski, AN., Lin, DW., Boskey, AL., Donnelly, E., Estroff, LA., Butcher, JT.. (2018). Crystallinity of hydroxyapatite drives myofibroblastic activation and calcification in aortic valves. Acta Biomaterialia. 71, 24-36. https:// doi.org/10.1016/j.actbio.2018.02.024

9. Lamichhane, S., Anderson, J., Remund, T., Kelly, P., Mani., G. (2016). Dextran sulfate as a drug delivery platform for drugcoated balloons: Preparation, characterization, in vitro drug elution, and smooth muscle cell response. Journal of Biomedical Materials Research. Part B, Applied Biomaterials, 104(7), 1416-30. bttps:// doi.org/10.1002/jbm.b.33494

10. Lin, W. C., Yu, D. G., \& Yang, M. C. (2005). pH-sensitive polyelectrolyte complex gel microspheres composed of chitosan/sodium tripolyphosphate/dextran sulfate: Swelling kinetics and drug delivery properties. Colloids and Surfaces B: Biointerfaces, 44(2-3), 143-151. https:/ / doi.org/10.1016/j.colsurfb.2005.06.010

11. Van Tomme, S. R., \& Hennink, W. E. (2007). Biodegradable dextran hydrogels for protein delivery applications. Expert Review of Medical Devices. https://doi.org/10.1586/17434440.4.2.147 
12. Arturson, G., \& Wallenius, G. (1964). The intravascular persistence of dextran of different molecular sizes in normal humans. Scandinavian Journal of Clinical and Laboratory Investigation, 16(1), 76-80. https:/ / doi.org/10.3109/00365516409060485

13. Sarmento, B., Ribeiro, A., Veiga, F., \& Ferreira, D. (2006). Development and characterization of new insulin containing polysaccharide nanoparticles. Colloids and Surfaces B: Biointerfaces, 53(2), 193-202. https:/ / doi.org/ 10.1016/j.colsurfb.2006.09.012

14. Van Tomme, S. R., Van Steenbergen, M. J., De Smedt, S. C., Van Nostrum, C. F., \& Hennink, W. E. (2005). Self-gelling hydrogels based on oppositely charged dextran microspheres. Biomaterials, 26(14), $2129-2135$. https:// doi.org/10.1016/j.biomaterials.2004.05.035

15. Hofbauer, LC., Brueck, CC., Shanahan, CM., Schoppet, C., Dobnig, H. (2007). Vascular calcification and osteoporosis--from clinical observation towards molecular understanding. Osteoporosis International: A Journal Established as Result of Cooperation between the European Foundation for Osteoporosis and the National Osteoporosis Foundation of the USA, 18(3), 251-9. bttps: / / doi.org/ 10.1007/s00198-006-0282-z

16. Hofbauer, LC., \& Schoppet, M. (2004). Clinical implications of the osteoprotegerin/RANKL/RANK system for bone and vascular diseases. JAMA. https:// doi.org/10.1001/jama.292.4.490

17. Steeve, KT., Marc, P., Sandrine, T., Dominique, H., \& Yannick, F. (2004). IL-6, RANKL, TNF-alpha/IL-1: Interrelations in bone resorption pathophysiology. Cytokine and Growth Factor Reviews. https:/ / doi.org/ 10.1016/j.cytogfr.2003.10.005

18. Sandberg, WJ., Yndestad, A., Øie, E., Smith, C., Ueland, T., Ovchinnikova, O., Robertson, A., Müller, F., Semb, AG., Scholz, H., Andreassen, AK., Gullestad, L., Damås, JK., Frøland, SS., Hansson, GK., Halvorsen, B., Aukrust, P. (2006). Enhanced Tcell expression of RANK ligand in acute coronary syndrome: Possible role in plaque destabilization. Arteriosclerosis, Thrombosis, and Vascular Biology, 26(4), 857-863. https:// doi.org/10.1161/01.ATV.0000204334.48195.6a

19. Bucay, N., Sarosi, I., Dunstan, C. R., Morony, S., Tarpley, J., Capparelli, C., ... Simonet, W. S. (1998). Osteoprotegerin-deficient mice develop early onset osteoporosis and arterial calcification. Genes and Development, 12(9), 1260-1268. https:// doi.org/10.1101/gad.12.9.1260

20. Panizo, S., Cardus, A., Encinas, M., Parisi, E., Valcheva, P., López-Ongil, S., Coll, B., Fernandez, E., Valdivielso, JM. (2009). RANKL increases vascular smooth muscle cell calcification through a rank-bmp4-dependent pathway. Circulation Research, 104(9), 1041-1048. https:/ / doi.org/10.1161/CIRCRESAHA.108.189001

21. Biscetti, F., Giovannini, S., Straface, G., Bertucci, F., Angelini, F., Porreca, C., Landolfi, R., Flex, A. (2016). RANK/RANKL/OPG pathway: genetic association with history of ischemic stroke in Italian population. Eur Rev Med Pharmacol Sci, 20(21), 4574-4580. bttp:// mww.ncbi.nlm.nih.gov/pubmed/27874938

22. Khosla, S. (2001). Minireview: The OPG/RANKL/RANK system. Endocrinology. https://doi.org/10.1210/endo.142.12.8536

23. Engels, G. E., Blok, S. L. J., \& van Oeveren, W. (2016). In vitro blood flow model with physiological wall shear stress for hemocompatibility testing-An example of coronary stent testing. Biointerphases, 11(3), 31004. bttps://doi.org/10.1116/1.4958979

24. Asri, R. I. M., Harun, W. S. W., Hassan, M. A., Ghani, S. A. C., \& Buyong, Z. (2016). A review of hydroxyapatite-based coating techniques: Sol-gel and electrochemical depositions on biocompatible metals. Journal of the Mechanical Behavior of Biomedical Materials. https:// doi.org/10.1016/j.jmbbm.2015.11.031

25. Haddow, D. B., James, P. F., \& Van Noort, R. (1996). Characterization of sol-gel surfaces for biomedical applications. Journal of Materials Science: Materials in Medicine, 7(5), 255-260. https:// doi.org/10.1007/BF00058562

26. Gupta, R., \& Kumar, A. (2008). Bioactive materials for biomedical applications using sol-gel technology. Biomedical Materials (Bristol, England), 3(3), 34005-34020. https:// doi.org/10.1088/1748-6041/3/3/034005

27. Kim, J., Kim, I. S., Cho, T. H., Lee, K. B., Hwang, S. J., Tae, G., Noh, I., Lee, SH., Park, Y., Sun, K. (2007). Bone regeneration using hyaluronic acid-based hydrogel with bone morphogenic protein-2 and human mesenchymal stem cells. Biomaterials, 28(10), 1830-1837. https://doi.org/10.1016/j.biomaterials.2006.11.050

28. Dalton, PD., \& Woodfi, T. (2008). Polymeric Scaffolds for Bone Tissue Engineering. Bone, 32(3), $2004-2005$. bttps:// doi.org/10.1002/jbm.a.31829.ELECTROSPINNING

29. Dennis, S. C., Whitlow, J., Detamore, M. S., Kieweg, S. L., \& Berkland, C. J. (2017). Hyaluronic-Acid-Hydroxyapatite Colloidal Gels Combined with Micronized Native ECM as Potential Bone Defect Fillers. Langmuir: The ACS Journal of Surfaces and Colloids, 33(1), 206-218. https:/ / doi.org/10.1021/acs.langmuir.6b03529

30. Aarnoudse, W., van't Veer, M., Pijls, N. H. J., ter Woorst, J., Vercauteren, S., Tonino, P., ... van de Vosse, F. (2007). Direct Volumetric Blood Flow Measurement in Coronary Arteries by Thermodilution. Journal of the American College of Cardiology, 50(24), 2294-2304. https:// doi.org/10.1016/j.jacc.2007.08.047

31. Schorn, I., Malinoff, H., Anderson, S., Lecy, C., Wang, J., Giorgianni, J., \& Papandreou, G. (2017). The LUTONIX® drugcoated balloon: A novel drug delivery technology for the treatment of vascular disease. Advanced Drug Delivery Reviews. bttps://doi.org/10.1016/j.addr.2017.05.015

32. Kariya, Y., Honma, M., Aoki, S., Chiba, A., \& Suzuki, H. (2009). Vps33a mediates RANKL storage in secretory lysosomes in osteoblastic cells. Journal of Bone and Mineral Research, 24(10), 1741-1752. https:// doi.org/10.1359/jbmr.090409 


\section{ABOUT STUDENT AUTHORS}

Jenna Mosier, Hannah Stealey, Kalifa Stringfield, and Katie Webb graduated in May of 2018 from Mississippi State University.

\section{PRESS SUMMARY}

Vascular calcification is a condition in which the arteries stiffen and plaque build-up occurs, blocking blood flow. Current treatments for this issue are only temporary solutions, with drug-eluting stents being the most successful. This experiment seeks to design a gel coating for stents that is safe and nontoxic within the body and can successfully carry and release anti-calcification drugs to the state of diseased arteries. From the results, it was determined that a stable and biocompatible gel was formed. 